\title{
Salmonella enterica I 4,[5],12:i:- Associated with Lesions Typical of Swine Enteric Salmonellosis
}

\section{Bailey L. Arruda, Eric R. Burrough, Kent J. Schwartz}

Salmonella enterica serotype I 4,[5],12:i:- has been increasingly isolated from swine. However, its pathogenic potential is not well characterized. Analysis of swine cases confirmed a strong positive association between isolation of । 4,[5],12:i:- and lesions of enteric salmonellosis and suggested a similar pathogenic potential as that for Salmonella Typhimurium.

$\mathrm{O}$ ver the past decade, Salmonella enterica serotype 4,[5],12:i:- has emerged as a major public health threat in Europe (1) and the United States (2). As a monophasic variant of Salmonella Typhimurium, Salmonella I 4,[5],12:i:- was rarely identified before the mid-1990s (3). However, it has now been detected in cattle (4), poultry (5), and swine (4-6), and several human disease outbreaks associated with contaminated pork products have occurred $(7-10)$.

Salmonellosis is also a major disease concern in swine. Salmonella Typhimurium has been considered the most common cause of enteric salmonellosis in swine (11). Recent reports from 2 of the largest veterinary diagnostic laboratories in the United States showed that there was a noted increase in the percentage of isolates of Salmonella I 4,[5],12:i:- $(4,6)$.

Despite the apparent increase in the isolation of Salmonella I 4,[5],12:i:- from swine and pork products, there is currently limited information regarding the pathogenicity of this serotype in swine. Accordingly, this study assessed the pathogenic potential of Salmonella I 4,[5],12:i:- through the evaluation of microscopic intestinal lesions in swine enteric cases from which Salmonella I 4,[5],12:i:- was isolated compared with similar cases from which Salmonella I 4,[5],12:i:- was not isolated.

\section{The Study}

The Iowa State University-Veterinary Diagnostic Laboratory (ISU-VDL) is a National Animal Health Laboratory

Author affiliation: Iowa State University, Ames, lowa, USA

DOI: https://doi.org/10.3201/eid2507.181453
Network-accredited laboratory that receives $>75,000$ case submissions annually, of which most are derived from swine production systems located throughout the United States. Histopathologic analysis is performed by veterinary diagnostic pathologists on $\approx 10,000$ cases from swine per year. The ISU-VDL laboratory information management system provided the initial dataset for this analysis.

To determine whether isolation of Salmonella I 4,[5],12:i:- from samples submitted from pigs was associated with microscopic lesions consistent with enteric salmonellosis, we compared cases from which Salmonella I 4,[5],12:i:- was isolated with cases from which neither Salmonella I 4,[5],12:i:- nor Salmonella Typhimurium were isolated; these samples were collected during July 2016-December 2017. We also reviewed cases from which Salmonella Typhimurium or 1 of 3 Salmonella serogroup B serovars (Salmonella Derby, Salmonella Agona, and Salmonella Heidelberg) were isolated to determine the potential comparative pathogenicity of Salmonella I 4,[5],12:i:-. All of these cases met the following qualifying criteria: animals were 3-13 weeks of age, a Salmonella serovar as outlined above was isolated by direct culture performed on enteric tissues, and histopathologic analysis was performed on the large intestine. To determine the association between the presence of Salmonella I 4,[5],12:i:- and lesions consistent with enteric salmonellosis, we also reviewed 40 additional cases that met the previously stated inclusion criteria but from which neither Salmonella I 4,[5],12:i:- nor Salmonella Typhimurium were isolated; we randomly selected these cases from an Excel (Microsoft, https://www.microsoft. com) data file by using the RAND() function.

Enteric samples submitted for isolation of Salmonella were routinely processed and reported (Appendix, https:// wwwnc.cdc.gov/EID/article/25/7/18-1453-App1.pdf). Microscopic lesions deemed compatible with salmonellosis in sections of the large intestine included erosion; ulceration; neutrophilic infiltration; crypt ectasia, crypt elongation, or both with associated neutrophilic exudation; goblet cell loss; luminal accumulation of neutrophils and fibrin; and submucosal accumulation of lymphocytes and macrophages. We used JMP Pro 14 (SAS Institute, https://www.sas.com) to perform all analyses. We used the Pearson $\chi^{2}$ test and odds 
ratios to determine the association between isolation of $\mathrm{Sal}$ monella I 4,[5],12:i:- and pathologic diagnosis of enteric salmonellosis. We considered a $p$ value $\leq 0.05$ significant.

We isolated Salmonella I 4,[5],12:i:- from 138 porcine cases that met all of the qualifying criteria. We isolated Salmonella Typhimurium from 18 cases, and other potentially lesser pathogenic Salmonella serogroup B serovars, including Salmonella Derby, Agona, and Heidelberg, from 35 cases.

A review of case data for clinical submissions to the ISU-VDL confirmed a statistically significant positive association between histologic lesions consistent with enteric salmonellosis and isolation of Salmonella I 4,[5],12:i:(odds ratio 10.53, 95\% CI 4.45-24.88; $\mathrm{p}<0.0001$ ) (Table). We confirmed compatible histologic lesions of salmonellosis (Figure) for 100 (72\%) of 138 cases from which Salmonella I 4,[5],12:i:- were isolated (Appendix Table 1). Histologic lesions consistent with enteric salmonellosis from which neither Salmonella I 4,[5],12:i:- nor Salmonella Typhimurium were isolated were observed for $8(20 \%)$ of 40 cases (Appendix Table 2).

Salmonella was isolated from 6 of the 40 cases, 3 of which had lesions consistent with enteric salmonellosis. We confirmed compatible histologic lesions of salmonellosis in 17 (94\%) of 18 cases in which Salmonella Typhimurium was isolated and 11 (31\%) of 35 cases in which another serogroup B Salmonella was isolated. Other agents of enteric disease that were concurrently detected in some cases included rotaviruses, coronaviruses, coccidians, and hemolytic Escherichia coli. However, none of these agents caused lesions consistent with those used to define salmonellosis in this report (Appendix Tables 1,2).

\section{Conclusions}

During an 18-month period and using the same qualifying criteria for cases, we identified Salmonella I 4,[5],12:i:from 138 swine cases. However, Salmonella Typhimurium was isolated from only 18 cases and another serogroup B Salmonella as specified above was isolated from only 35 cases. This finding represents nearly an 8-fold increase in isolation of Salmonella I 4,[5],12:i:- compared with Salmonella Typhimurium and is in concordance with findings of others who have identified an increase in isolation of Salmonella I 4,[5],12:i:- from swine samples submitted to veterinary diagnostic laboratories $(4,6)$.

A major aspect of Salmonella epidemiology is the variation in prevalence of serotypes or phage types over time in human and animal populations. The catalysts of such changes remain elusive (3). Isolation of Salmonella I 4,[5],12:i:- was rarely reported before 1993 (3), but this serotype has now become predominant in human clinical cases and has been isolated from food products, including pork, on different continents (3).
Table. Diagnostic cases with and without colitis in swine and isolation of Salmonella enterica I 4,[5],12:i:-*

\begin{tabular}{lcc}
\hline & \multicolumn{2}{c}{ Colitis lesion } \\
\cline { 2 - 3 } $\mid 4,[5], 12: i:-$ isolated & No & Yes \\
\hline No & 32 & 8 \\
Yes & 38 & 100 \\
\hline${ }^{*}$ Odds ratio $10.5(95 \% \mathrm{Cl}$ & $4.45-24.88) ; p<0.0001$ by Pearson $\chi^{2}$ test. \\
\hline
\end{tabular}

Although increased isolation of Salmonella I 4,[5],12:i:- from swine samples has been documented, the pathogenic potential of this serovar in pigs had not been reported. In this study, we demonstrate a strong positive association between histologic lesions consistent with enteric salmonellosis and isolation of Salmonella I 4,[5],12:i:-. In most cases from which Salmonella I 4,[5],12:i:- or Salmonella Typhimurium were isolated, the severity of histologic lesions was similar. However, the percentage of cases in which histologic lesions consistent with enteric salmonellosis were present was lower for cases from which Salmonella I 4,[5],12:i:- (72\%) was isolated than for cases from which Salmonella Typhimurium (94\%) was isolated. Based on these data, we believe that Salmonella I 4,[5],12:i:- is a likely cause of enteric salmonellosis that has a similar or perhaps slightly lower pathogenic potential in swine than Salmonella Typhimurium. Pathogenicity studies are needed to further characterize the pathogenic potential and

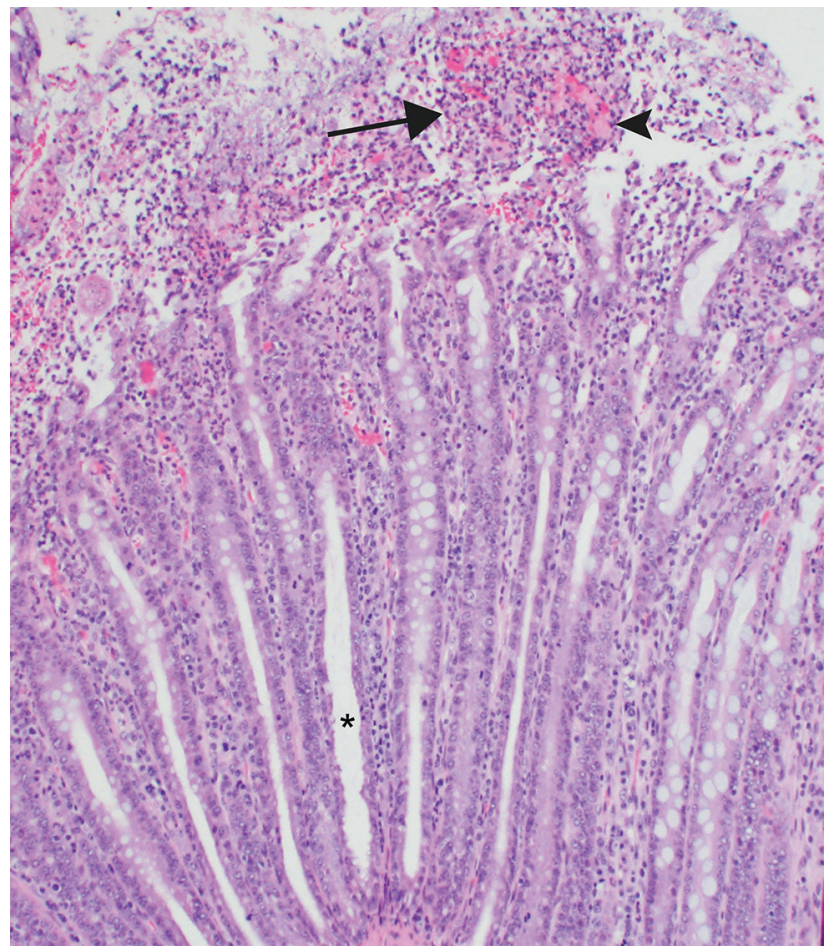

Figure. Section of large intestine from a pig infected with Salmonella enterica subsp. enterica serotype I 4,[5],12:i:-. Asterisk indicates crypt elongation and goblet cell loss, arrow indicates abundant degenerate neutrophils in the lumen, and arrowhead indicates abundant fibrin in the lumen. Hematoxylin and eosin stain; original magnification $\times 100$. 
fitness of Salmonella I 4,[5],12:i:- compared with that of Salmonella Typhimurium in swine.

We suspect that Salmonella I 4,[5],12:i:- has evolutionary advantages that have resulted in its predominance as one of the most common Salmonella serotypes responsible for swine enteric salmonellosis. Accordingly, it is essential to determine the putative attributes that facilitate its rapid spread and ecologic success. Specifically, antimicrobial drug resistance genes and genes that encode resistance to heavy metal micronutrients should be evaluated, given their current and common use in US swine production.

\section{About the Author}

Dr. Arruda is an assistant professor and diagnostic pathologist at the Iowa State University Veterinary Diagnostic Laboratory, Ames, IA. Her primary research interest is infectious diseases of swine.

\section{References}

1. European Food Safety Authority. The European Union summary report on trends and sources of zoonoses, zoonotic agents and food-borne outbreaks in 2012. EFSA J. 2014;12:3547 [cited 2019 Mar 11]. https://www.efsa.europa.eu/en/efsajournal/pub/3547

2. Centers for Disease Control and Prevention. Outbreak of multidrug-resistant Salmonella I 4,[5],12:i:- infections linked to pork; 2015 [cited 2018 Jul 30]. http://www.cdc.gov/salmonella/ pork-08-15/index.html

3. Switt AI, Soyer Y, Warnick LD, Wiedmann M. Emergence, distribution, and molecular and phenotypic characteristics of Salmonella enterica serotype 4,5,12:i-. Foodborne Pathog Dis. 2009;6:407-15. http://dx.doi.org/10.1089/fpd.2008.0213

4. Hong S, Rovira A, Davies P, Ahlstrom C, Muellner P, Rendahl A, et al. Serotypes and antimicrobial resistance in Salmonella enterica recovered from clinical samples from cattle and swine in Minnesota, 2006 to 2015. PLoS One. 2016;11:e0168016. http://dx.doi.org/10.1371/journal.pone.0168016

5. Centers for Disease Control and Prevention. National Antimicrobial Resistance Monitoring System: Retail Meat Interim Report; 2016 [cited 2018 Jul 30]. https://www.fda.gov/downloads/ AnimalVeterinary/SafetyHealth/AntimicrobialResistance/National AntimicrobialResistanceMonitoringSystem/UCM498134.pdf

6. Yuan C, Krull A, Wang C, Erdman M, Fedorka-Cray PJ, Logue CM, et al. Changes in the prevalence of Salmonella serovars associated swine production and correlations of avian, bovine and swine-associated serovars with human-associated serovars in the United States (1997-2015). Zoonoses Public Health. 2018;65:64861. http://dx.doi.org/10.1111/zph.12473

7. Bone A, Noel H, Le Hello S, Pihier N, Danan C, Raguenaud ME, et al. Nationwide outbreak of Salmonella enterica serotype 4,12:i:0- infections in France, linked to dried pork sausage, March-May 2010. Euro Surveill. 2010;15:19592.

8. Mossong J, Marques P, Ragimbeau C, Huberty-Krau P, Losch S, Meyer G, et al. Outbreaks of monophasic Salmonella enterica serovar 4,[5],12:i:- in Luxembourg, 2006. Euro Surveill. 2007;12:E11-2. http://dx.doi.org/10.2807/esm.12.06.00719-en

9. Hauser E, Tietze E, Helmuth R, Junker E, Blank K, Prager R, et al. Pork contaminated with Salmonella enterica serovar 4,[5],12:i:-, an emerging health risk for humans. Appl Environ Microbiol. 2010;76:4601-10. http://dx.doi.org/10.1128/AEM.02991-09

10. Imanishi M, Anderson TC, Routh J, Brown C, Conidi G, Glenn L, et al. Salmonellosis and meat purchased at live-bird and animal-slaughter markets, United States, 2007-2012. Emerg Infect Dis. 2014;20:167-9. http://dx.doi.org/10.3201/eid2001.131179

11. Carlson SA, Barnhill AE, Griffith RW. Salmonellosis. In: Zimmerman JJ, Karriker LA, Ramirez A, Schwartz KJ, Stevenson GW, editors. Disease of swine. Oxford: John Wiley \& Sons, Inc; 2012. p. 821-31.

Address for correspondence: Bailey L. Arruda, Department of Veterinary Diagnostic and Production Animal Medicine, Iowa State University, 1850 Christensen Dr, Ames, IA 50011, USA; email: wilberts@iastate.edu 